\title{
Editorial
}

\section{Biobased Polymers and Composites}

\author{
Chaoqun Zhang $\mathbb{D}^{1}{ }^{1}$ Rafael L. Quirino, ${ }^{2}$ and Jianzhong Sun $^{3}$ \\ ${ }^{1}$ College of Materials and Energy, South China Agricultural University, Guangzhou 510642, China \\ ${ }^{2}$ Department of Chemistry, Georgia Southern University, Statesboro, GA 30460, USA \\ ${ }^{3}$ Biofuels Institute, School of the Environment, Jiangsu University, Zhenjiang 212013, China
}

Correspondence should be addressed to Chaoqun Zhang; nwpuzcq@gmail.com

Received 28 June 2018; Accepted 28 June 2018; Published 15 July 2018

Copyright ( 92018 Chaoqun Zhang et al. This is an open access article distributed under the Creative Commons Attribution License, which permits unrestricted use, distribution, and reproduction in any medium, provided the original work is properly cited.

With the worldwide depletion of crude oil stock and increasing environmental concerns, efforts on a global scale are dedicated to developing viable alternatives to conventional petroleum-based polymeric materials for a sustainable and green society. Renewable resources, such as vegetable oils, cellulose, starch, and lignin are promising options, which are abundantly produced by nature through photosynthesis from carbon dioxide and water. Innovative technologies to transform these natural resources into value-added chemicals and novel polymerization methods for production of high-performance and low-cost polymers with tunable structures and functionalities are key parts of sustainable development. In addition, the development of state-of-theart characterization techniques for these new materials is an essential component in uncovering their specific structures and facilitates the application of these materials in new areas, such as sensors, structural parts, and biomedical devices.

Despite the progress made in renewable polymers, such as PLA, in the last decades, most of the proposed biobased materials are far from commercial application and from replacing petroleum-based products. In order to improve physical and thermomechanical properties, nanofillers and fibers have been incorporated into biobased polymer matrices. This special issue aims at providing a great platform for scientists and researchers in the area of biobased polymers and composites to communicate their state-of-the-art work related to the transformation of natural resources into value-added chemicals, the modification and preparation of new bio-based polymers, and the characterization and application of biobased polymers and composites. In this special issue, original research and review articles that uncover the development of biobased polymers and composites are collected, including new technologies related to their synthesis, processing, characterization, and application. The topics emphasized in this special issue include the following:

(1) Biobased polymers synthesized from natural feedstock

(2) Bionanocomposites with renewable reinforcements

(3) Fiber-reinforced polymers with biobased matrix or biobased fibers

(4) Processing and modification of natural macromolecules

(5) Specific properties and new application of biobased polymers and their composites

\section{Acknowledgments}

We would like to express our appreciation to all the authors for their informative contributions and the reviewers for their strong support and constructive critiques in making this special issue possible.

Chaoqun Zhang

Rafael L. Quirino

Jianzhong Sun 


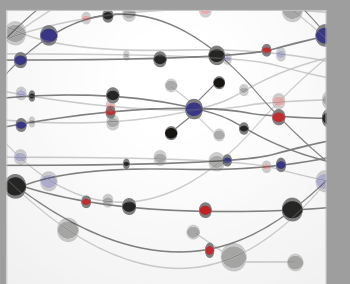

The Scientific World Journal
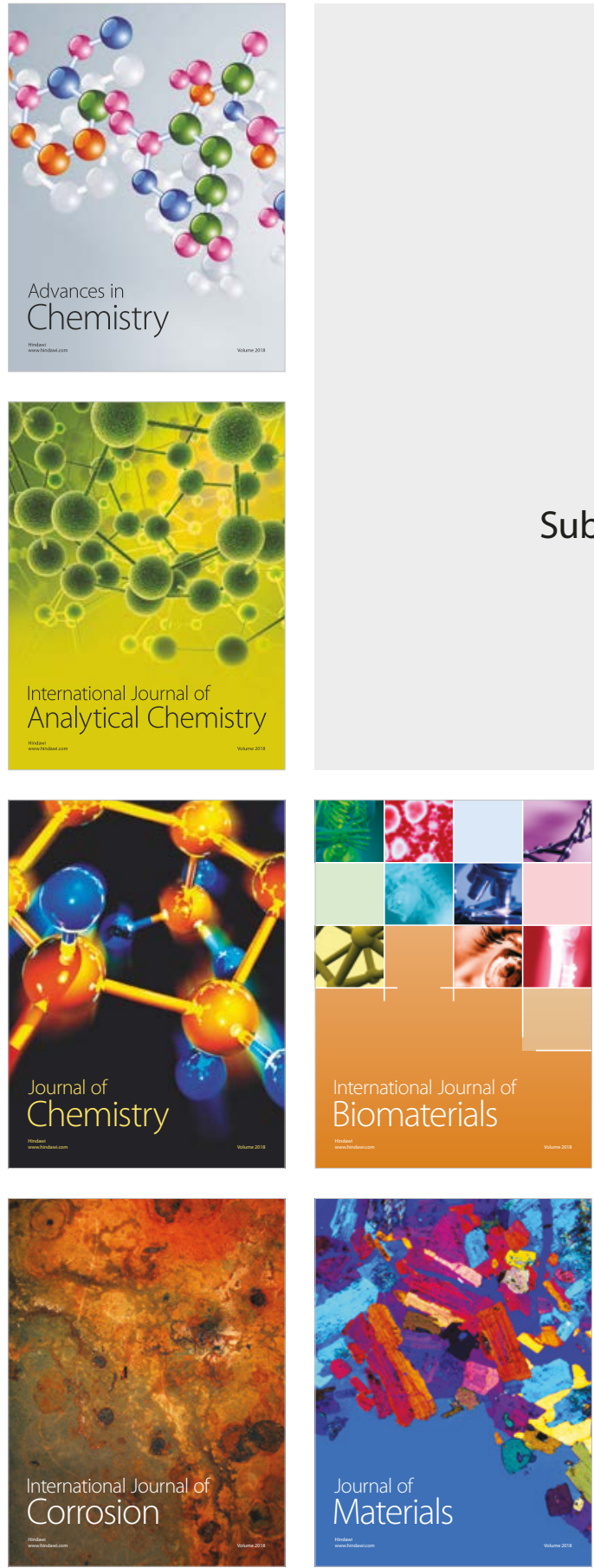

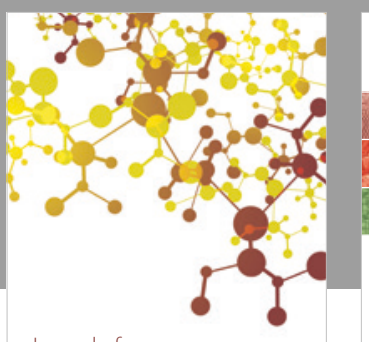

Journal of

Applied Chemistry
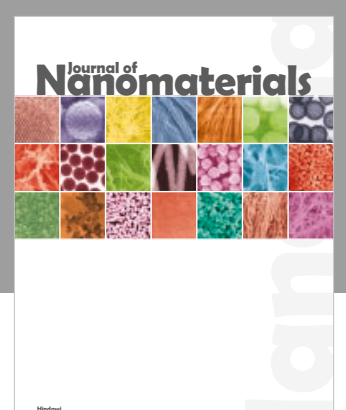

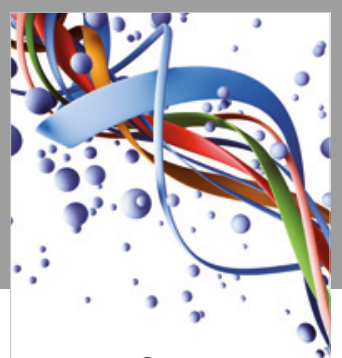

Scientifica

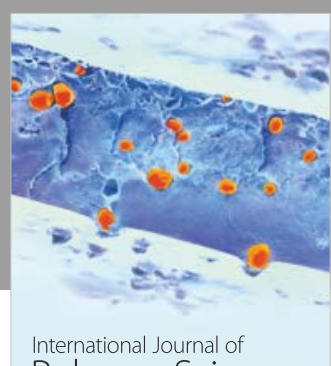

Polymer Science

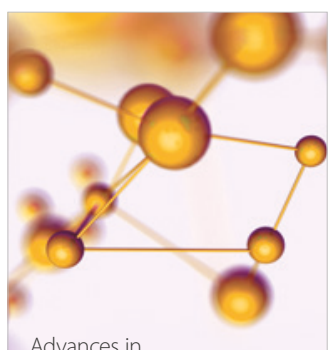

Physical Chemistry
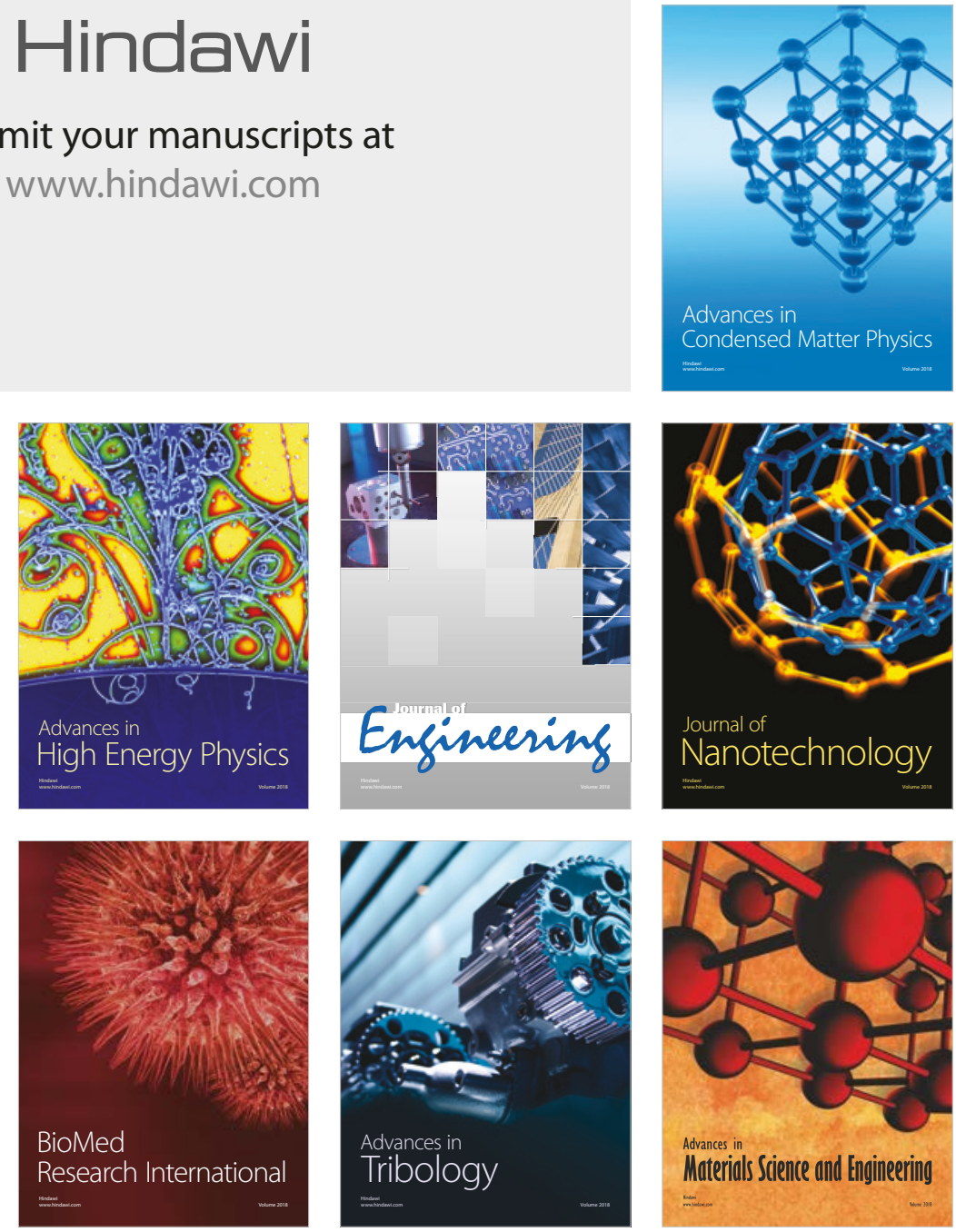